\title{
Initial Studies in Human-Robot-Human Interaction: Fitts' Law for Two People
}

\author{
Kyle Reed, Michael Peshkin, J. Edward Colgate, James Patton \\ Laboratory for Intelligent Mechanical Systems \\ Northwestern University \\ Evanston, Illinois 60208 \\ \{reedkb, peshkin, colgate, j-patton\}@northwestern.edu
}

\begin{abstract}
Often two people must work together physically on a common task, such as lifting and positioning a long board, or, in our model experimental system, turning a two-handled crank. Such tasks involve communication between the people, mediated by the task kinematics and dynamics: each person feels forces and motions produced by the other and derives some meaning from them. Tasks may include a degree of competition: the two people may not have exactly the same goal in mind, and must negotiate a compromise. Understanding human-human communication is important in designing robots for interaction with humans, and for robots that provide powered assistance for human-human tasks (such as physical therapy).
\end{abstract}

In this paper we describe early experiments in human-human physical interaction, with a 1 dof robot included in order to give experimental access to the exchange of forces and motions between the people.

We report on Fitts' law-like tasks, in which the two people cooperate to move a cursor to a common target, or to targets that do not completely overlap. Our results suggest that humanhuman physical communication may be a rich area of study.

Human-robot-human, human-human, haptic interaction, Fitts' law

\section{MOTIVATION}

In numerous contexts, two people share physical tasks, and communicate through their physical interaction. Imagine two people working together to move a mattress through a doorway and up a flight of stairs. Each person is interacting with the other person through the object, each with his or her own forces and thoughts about where they are going. Similarly, imagine people exchanging a glass of water without spilling a drop of it. Some airplanes and helicopters have a flight stick that is coupled between the pilot and co-pilot. When both the pilot and co-pilot are holding the handles, they are working together and communicating through these handles. In each of these examples, physical cues are passed from one person to the other.

It stands to reason that homo sapiens, a social species, has had opportunity to develop sophisticated ways of sharing a physical task, involving communicating with each other physically, compromising with respect to task goals, and teaching and learning manual skills. We expect that a significant channel of dyad communication should be through forces and motions, applied either directly to one another's limbs, or via a mutually grasped object.

Our motivating area of interest, lower limb rehabilitation, also involves haptic interaction between two people. The hands-on interaction between physical therapist and patient involves the communication of muscle tone, force, and motion to the patient, and the selective delivery of force and motion by the therapist.

These examples suggest basic scientific questions in an area which we denote "human-robot-human" (HRH) interaction. We are exploring how two people physically cooperate, compromise, and guide one another, through force and motion, and how machine-generated forces and motions can enter into the human-human physical conversation.

\section{RELATED WORK}

Lifting and moving a table cannot be done individually, so people work together to accomplish this task. Takubo et al. [1] demonstrate how a robot can assist a person moving a table. The human is dominant and leads while the robot emulates a virtual non-holonomic constraint to keep it passively following. It is not known how two humans perform this same task. Learning how two humans communicate haptically through a rigid link will help in creating more intuitive communication between a robot and a human.

Yanco and Drury [2] discuss many different combinations of how humans and robots can work together. All of their classifications are based on one or many humans giving an order to one or many robots and the robots doing the task. They do not classify the situation in which there are two humans giving an order to a robot that is acting on those same humans.

Klingsport et al. [3] discuss various methods for communication between humans and robots, including programming commands, listening to natural words, and demonstration. They aim to make it as easy and intuitive as possible for people to work with a robot. To make it easy, human-robot communication in a shared task should follow the implicit human-human communication standards, which are poorly understood.

Sallnas and Zhai [4] performed a study with a haptic system simulating the handoff of an object. They measured the time it 
takes to hand off objects within certain targets. Here the two people are not physically connected, they feel only the sensations the haptic device can simulate.

Learning a new sport, such as tennis, is often performed by an expert guiding a new student through the motions. Can the learning rate be increased if the teacher and student have different authority over the intended motion? In one scenario, the student will have no influence in the beginning and as his skills increase, he will be allowed more control until the student has complete control and the teacher has no influence. Gillespie et al. [5] demonstrates a student being taught by a virtual teacher. The virtual teacher operates much like the tennis instructor, moving the student's hand through a motion, while also permitting some feel of the task. In this study, the virtual teacher is either present or not - graduated influence is not explored.

Wegner and Zeaman [6] did studies on the effects of learning in groups of 2 and 4 compared to doing a task individually. They found that working in groups does help in learning a task, with the effectiveness decreasing as the size of the group increases.

Both Elhajj et al. [7] and Hespanha et al. [8] have explored two humans communicating in a teleoperational haptic environment over the internet. Barnes and Counsell [9] explore these same issues, but in a physically nearby location, much like Sallnas and Zhai's setup with the handoff computer simulation, [4]. In each of these cases, the two people are not physically or rigidly connected.

Rahman et al. [10] have worked on modeling the impedance characteristics of a human arm showing the resistance an arm will apply when it is led through a given path. They discuss two humans interacting, but only make mention of the fact that one of the humans will be the leader and the other will be the follower in a cooperative task. Rahman et al. [11] then take the characteristics of a human response found in their studies and implement the same response in a robot. Their aim is to make the robot imitate the response of a human when interacting with another human.

Bruning et al. [12] did a comparison between the performance of two people working cooperatively or competitively. They found that competition led to faster results.

Kumar et al. [13] shows preliminary studies of a device to extend a hand for fine motor work and force scaling.

\section{FITT'S LAW}

Fitts' law [14], described in 1954, is an empirical relation observing that the performance time for a person to move a distance $\mathrm{D}$ to a target of size $\mathrm{S}$ varies linearly with the "index of difficulty" ID, which is the logarithm of $\mathrm{D} / \mathrm{S}$, or $\mathrm{t}=\mathrm{a}+\mathrm{b} \log _{2}(\mathrm{D} / \mathrm{S})$, where $\mathrm{a}$ and $\mathrm{b}$ are constants. For a given target size, it takes longer to move a large distance than a small distance. It takes longer to move to a small target than to a larger target.

Over its long history, Fitts' law has proven to be remarkably robust. It has been observed in tasks of varying number of degrees of freedom and complexity, for instance: cursor movement along a line to a target line segment; cursor movement in a plane to a target disk, moving a can to a shelf, a peg into a hole, stringing beads, and many others [15]. The coefficients of Fitts' law vary from task to task (and to some extent from person to person), but the linearity in $\log (D / S)$ is observed over a wide range of index of difficulty, and over a wide variety of tasks.

Mottet et al. [15] show that Fitts' law applies to two people moving toward a moving target that is equally trying to reach them. An example of this is two people reaching to shake hands - person A's target, person B's hand, is equally trying to grab person A's hand, which is person B's target.

Fitts' law has been used in many areas of research from path tracking [16] to 3D computer games [17] to scrolling time on a computer [18] to GUI design [19]. However, Sallnas and Zhai [4] and Mottet et al. [15], are the only experiments we have been able to find on Fitts law for more than one person.

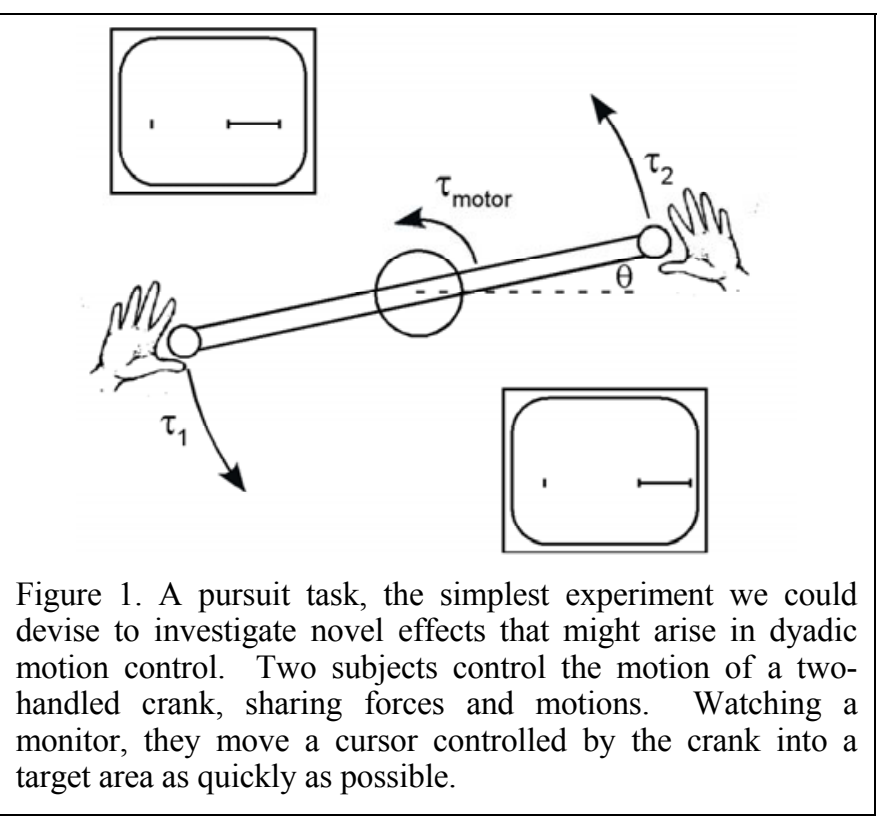

\section{Two PERSON FITTS' LAW}

As an initial foray into human-robot-human interaction, we tried to devise the simplest experiment that could reveal novel effects that arise in dyadic motion control. We chose a oneaxis motion configuration in which two subjects participate symmetrically. The task was also chosen to be as simple and conventional as possible: a one-axis pursuit task of the "Fitts' law" type.

Our experiment is an extension of the prototypical Fitts' law experiment. (Figure 1.) Two human subjects complete the task together. Each subject views a monitor, which shows a cursor and a target region. The cursor moves horizontally according to the angle $\theta$ of a two-handled crank, operating in the horizontal plane, with the handles held by the subjects, sitting opposite each other. When the cursor is successfully brought to and held in the target region, the target region moves 
to a new location. (The motor and the dual monitors will be explained later.)

We wish to determine how people work together and communicate physically and, specifically, whether Fitts' law describes the performance of two people engaged in a physically shared task with a common goal, or a task in which the two people have slightly divergent goals.

In this study, we look at how two individual efforts combine. Does Fitts' law hold true for two people sharing the same task? How does dyadic performance compare to individual performance?

Additionally, we are interested in negotiation and compromise through physical communication. Does Fitts' law hold true for two people with slightly different tasks in which reaching the goal requires coming to a physical compromise with each other?

We performed three experiments:

\section{Experiment 1: Individual pursuit}

In the first experiment, only one subject holds a crank handle. Each subject was given four sizes of targets at slightly varying distances D to determine each person's individual (one-person) Fitts' curve. This experiment reproduces one of many classic Fitts' law experiments.

\section{Experiment 2: Dyadic pursuit; common target}

In the second experiment, two subjects control the cursor together, coupled physically by the two-handled crank. Although they view separate monitors, the monitors show the same cursor and target, so that the subjects are working together physically with the same goal. Typical tasks as seen by the two subjects are shown in Figure 2. Since the inertia and friction of the device are small, there is little to get in the way of the haptic communication between the subjects. This experiment addresses physical communication with no conflict in goal, and in which no physical compromise is required.

\section{Experiment 3: Dyadic pursuit; differing target}

In the third experiment, two subjects control the cursor together, but the target seen by subject A does not entirely agree with the target seen by subject B. (Each subject can only see his/her own task.) Figure 3 shows one possible configuration. In this case, the two subjects must physically communicate and compromise to move the cursor to a position where both subjects are satisfied.

\section{EXPERIMENTAL APPARATUS \& PROTOCOL}

The apparatus is shown in Figure 4. The handles are connected via a rigid link that can spin freely at the center where a direct drive motor is attached (under the table), although the motor was not used in the experiments reported here. An optical encoder provides the rotational position of the bar. The monitors display identical images, but the task for the other person is covered up, so that each subject sees only his or her own cursor and target.

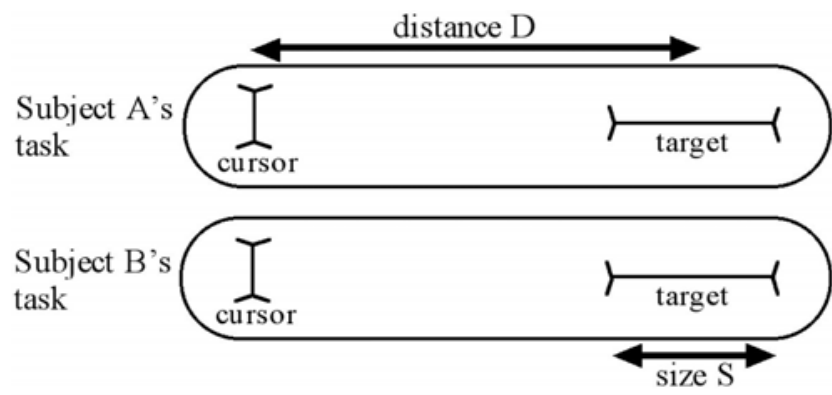

Figure 2. Identical targets for subjects A and B in experiment 2. Subjects physically cooperate to reach the common mutual goal. No physical compromise is required.

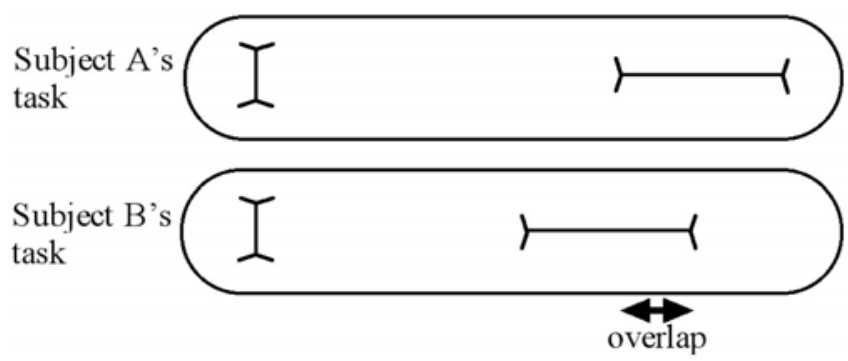

Figure 3. Divergent targets for subjects A and B in experiment 3. Subjects $\mathrm{A}$ and $\mathrm{B}$ control a cursor via the motion of the crank, but the target regions seen by the two subjects do not entirely agree. Thus the subjects must physically communicate and compromise in order for both of them to achieve their goals.

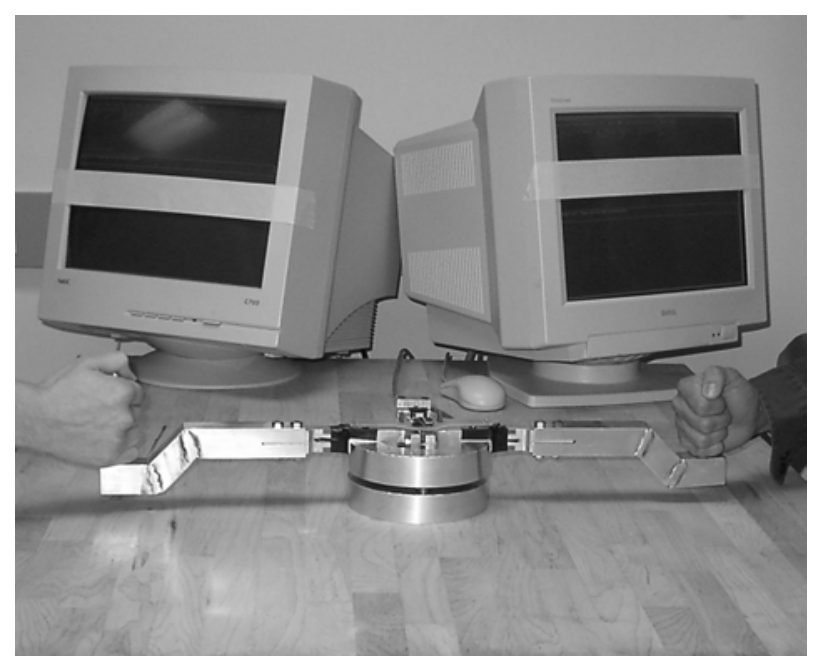

Figure 4. Experimental setup with two monitors and twohandled crank. The monitors display identical images, but the task for the other person is covered up, so that each subject sees only his or her own task. 
On each monitor a cursor, corresponding to the angular position of the handle, is presented to show the current location. Also shown is a target region. The target changes color when the cursor is within it. After the subject has held the handle in the target zone for one second, a new target will appear. The targets vary between one of four sizes: $2.4^{\circ}(1.3$ $\mathrm{cm}$ around the circle at the handle), $5.6^{\circ}(3.1 \mathrm{~cm}), 10.4^{\circ}(5.7$ $\mathrm{cm})$, or $16.8^{\circ}(9.2 \mathrm{~cm})$ with a distance between targets of $72^{\circ} \pm$ $8^{\circ}(30.2 \pm 4.4 \mathrm{~cm})$. In cases where the targets did not completely overlap, there was a minimum of $2.4^{\circ}(1.3 \mathrm{~cm})$ of overlap.

Each pair of subjects performed two rotations through the tasks. For each rotation, subjects did 24 targets individually (experiment 1), 24 targets together (experiment 2), and 60 targets together with various overlapping (experiment 3 ) for a total of 216 target matchings each. The first experiment forms a basis for each person's individual Fitts' law curve. The second experiment evaluates the performance of the same two subjects, now working in cooperation. The third experiment begins to study how physical compromise takes place. Verbal communication was not allowed at any point during the experiment. The sequence of experiments took about 20 minutes per pair of subjects.

\section{RESULTS}

We ran these experiments on 4 sets of subjects $(8$ subjects total). This data set is not large, but allows preliminary indications to direct future research. For the 8 individual subjects, the results (Figure 5) show a high correlation to Fitts' law $\left(\mathrm{R}^{2}=0.96\right)$. Each individual's average results are shown, as well as an average across subjects. Results are clustered along the ID axis because distance D was always similar, while target size $\mathrm{S}$ varied discretely among 4 values.

Figure 6 shows results when these same subjects worked together in dyads. On average, dyads shown identical targets were 0.14 seconds faster than individuals. The performance data for dyads also obeyed Fitts' law, with somewhat weaker correlation. $\left(\mathrm{R}^{2}=0.90\right)$.

When the two subjects have slightly different goals, we must clarify what we mean by "target size." It seems reasonable that the target size $\mathrm{S}$ should now be interpreted as overlap distance. However, as shown in Figure 7, we do not find a Fitts' law type behavior for this task. Since, historically, a very wide class of tasks have shown Fitts' law behavior, even for definitions of ID that are quite diverse and task-specific, our result is perplexing.

\section{DISCUSSION}

These are preliminary experiments, but they have produced several interesting results.

\section{Experiment 2: identical targets}

We observed significantly faster performance for dyads than for individuals. Movement of the crank is very easy, so it is not credible to us that the additional force capabilities of two people would be helpful. We find the result surprising because

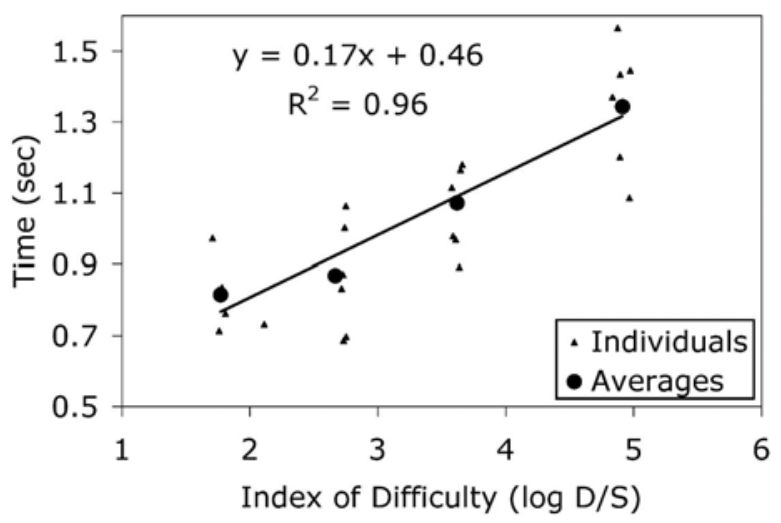

Figure 5. Results for experiment 1, in which one subject operated the crank. Average results are shown, as well as an average across subjects. Agreement with Fitts' law is seen.

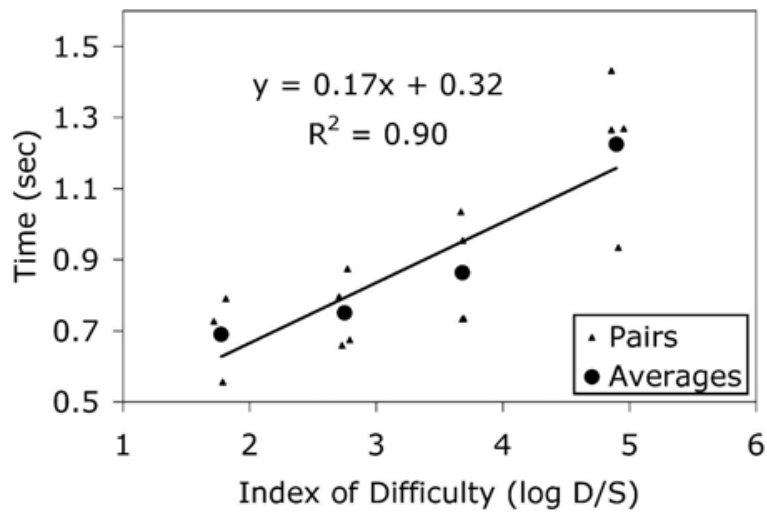

Figure 6. Results for experiment 2, in which two subjects operated the crank, and were shown identical target regions. Fitts law is observed, with an average performance time 0.14 seconds faster than for individuals.

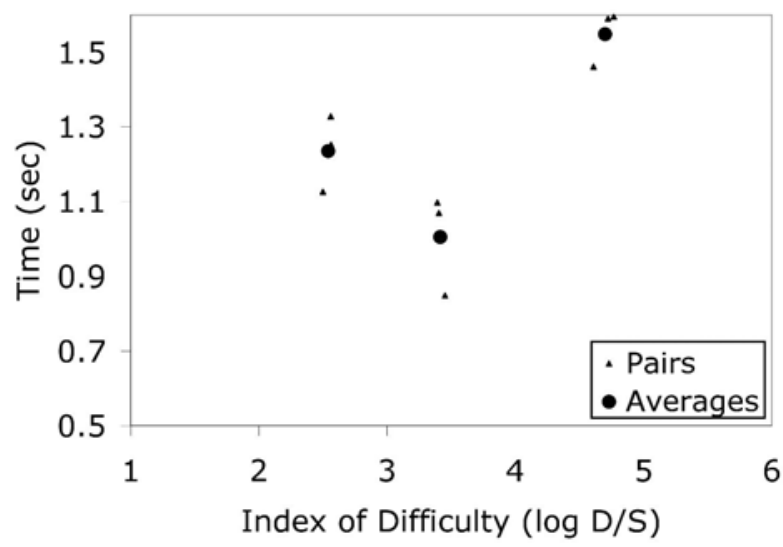

Figure 7. Results for experiment 3, in which two subjects operated the crank, and were shown overlapping, but different target regions, so that physical compromise was required. The performance time does not follow Fitts law. 
communication and compromise are required; the subjects can easily interfere with each other, and indeed some subjects reported frustration in working with a partner. (Our result is for a small number of subjects however, so further experimental verification and statistical analysis is called for.)

Some participants reported awareness that they had achieved what we will call specialization: one subject would take greater responsibility for the initial saccade to move toward the target region when it appeared, and the other would take greater responsibility for bringing the cursor to a stop accurately.

We suggest a physiologically reasonable (though speculative) opportunity for superior performance by a dyad. A single person executing the task in our preliminary experiment would be expected to use the so-called triphasic burst pattern of muscle activity, in which an agonist muscle burst starts the movement, and is followed by antagonist muscle burst to brake the movement and another agonist burst to help hold the limb at the final position [20][21][22][23]. These bursts represent careful planning based on prior knowledge, rather than feedback received during the task [24][25]. Moreover, these patterns represent optimal movements that best accomplish the task within rather limiting physiological constraints such as the rates at which muscles can be turned on and off [26][25] and the limited torque generating capacity in different areas of the workspace [27].

We hypothesize that when subjects are exposed to a dyadic task, they specialize so that one subject acts as the task's agonist and the other the antagonist, or as if one person is controlling the early phase (launch) and the other is controlling the late phase (braking). Consequently, the rate of force onset and offset becomes less critical if one person can be ramping up while the other is ramping down. Our preliminary anecdotal evidence suggests this type of specialization.

Changing from a one-person to a two-person task makes the system redundant, affording a new luxury of choices in how control is carried out [28]. There is no longer a one-to-one correspondence between dynamics and kinematics [29]. For example, in the dyadic tasks of our preliminary experiment, one subject can choose not to perform at all, to help with only the braking phase, or to use only elbow flexor muscles. One can imagine a variety of possibilities that did not exist in the one-person case that are advantageous. For example, cocontraction strategies similar to those used in parallel robotics and in human bimanual control [30] are possible in which both people share in a new form of "collaborative co-contraction" to stabilize the handle at the target.

\section{Experiment 3: differing targets}

The two-person/differing-target experiment does not seem to exhibit Fitts' law behavior, at least if we consider "target size" S to be the overlap distance, which seems to be the natural definition. In many cases, the two people would overcorrect and bounce back and forth around the target.

Shergill et al. [31] describe an experiment in which each of two subjects perceives that the other is pushing (or hitting) harder. In our cooperative task, if each subject feels that the other person is pushing harder, this could lead to poor performance since each has an exaggerated perception of the other's intent. If intent could be sensed more accurately, possibly by altering the amount of force conducted though the haptic interface, then perhaps the dyadic efficiency could be improved.

\section{VIII.FUTURE WORK}

We plan future experiments using the same or an enhanced experimental setup, motivated by the preliminary results discussed here. Our apparatus includes a direct drive motor, and strain gages on each handle. These features were not used in the experiments reported. In fact, because the crank mechanism is so light, the strain gages cannot differentiate the forces provided by one subject as opposed to the other.

\section{Specialization.}

By using the motor to create a simulated inertia, we can separately measure the forces and perhaps the roles of the two subjects. We plan to investigate the specialized roles that individuals adopt in a dyad, if indeed specialization is what is occurring.

\section{Adaptation.}

There is presumably an adaptation process during which subjects learn to move together effectively. The question of how adaptation takes place is independent of whether specialization is a general principle. How fast is the adaptation as a naïve dyad progresses through early trials together? After adapting as a part of one dyad, does a subject adapt more or less quickly to a new partner?

\section{The language of physical communication.}

If we can decode the language of physical communication between human subjects, we can prove our understanding by surreptitiously substituting the motor for the action of one of the subjects, and observing if the resulting "cyborg" dyad is fully as effective as the human-human dyad. A harsher test of our understanding is whether we can also substitute the motor successfully in place of one subject during the adaptation period.

\section{ACKNOWLEDGMENTS}

Kyle Reed thanks the National Science Foundation for supporting his study and research.

\section{REFERENCES}

[1] T. Takubo, H. Arai, and K. Tanie, "Human-robot cooperative handling using virtual nonholonomic constraint in 3-D space," Proceedings of 2000 IEEE/RSJ International Conference on Intelligent Robots and Systems, pp. 300-305, 2000.

[2] H. A. Yanco and J. L. Drury, "A taxonomy for human-robot interaction," Proceedings of the AAAI Fall Symposium on Human-Robot Interaction, AAAI Technical Report FS-02-03, Falmouth, MA, November 2002, pp. 111-119. 
[3] V. Klingsport, J. Demiris, and M. Kaiser, "Human-robot communication and machine learning," Applied Artificial Intelligence Journal 11, pp. 719-746, 1997.

[4] E. Sallnas and S. Zhai, "Collaboration meets Fitts' law: Passing virtual objects with and without haptic force feedback," in Proceedings of INTERACT 2003, IFIP Conference on HumanComputer Interaction, pp. 97-104.

[5] R. Gillespie, M. O'Modhrain, P. Tang, D. Zaretzy, and C. Pham, "The virtual teacher," ASME International Mechanical Engineering Conference and Exposition, Anaheim, CA, November 1998.

[6] N. Wegner and D. Zeaman, "Team and Individual Performance on a Motor Learning Task," Journal of General Psychology, 1956, Vol. 55, pp. 127-142.

[7] I. Elhajj, H. Hummert, N. Xi, and Y. Liu, "Real-time haptic feedback in internet-based telerobotic operation," IEEE International Conference on Robotics and Automation, San Francisco, May 2000.

[8] J. P. Hespanha, M. McLaughlin, G. S. Sukhatme, M. Akbarian, R. Garg, and W. Zhu, "Haptic collaboration over the internet," Fifth Phantom Users Group Workshop, Aspen, CO, October, 2000.

[9] D. P. Barnes and M. S. Counsell, "Haptic communication for remote mobile manipulator robot operations," American Nuclear Society, Proceedings of the 8th Topical Meeting on Robotics and Remote Systems, Pittsburgh, PA, April 1999.

[10] M. M. Rahman, R. Ikeura, and K. Mitzutani, "Impedance characteristics of human arm for cooperative robot," International Conference on Control, Automation and Systems, pp. 1455-1460, 2002.

[11] M. M. Rahman, R. Ikeura, and K. Mizutani, "Control characteristics of two humans in cooperative task and its application to robot control," Proceedings of 2000 International Conference on Industrial Electronics Control and Instrumentation, pp. 1773-1778, 2000.

[12] J.L. Bruning, D.K. Sommer, and B.R. Jones, "The Motivational Effects of Cooperation and Competition in the MeansIndependent Situation," Journal of Social Psychology, 1996, Vol. 68, pp. 269-274.

[13] R. Kumar, P. Kerkelman, P. Gupta, A. Barnes, P. S. Jensen, L. L. Whitcomb, and R. H. Taylor, "Preliminary experiments in cooperative human/robot force control for robot assisted microsurgical manipulation," In Proceedings of IEEE International Conference on Robotics and Automation, volume 1, pages 610-617, 2000.

[14] P. M. Fitts, "The information capacity of the human motor system in controlling the amplitude of movement," Journal of Experimental Psychology, 1954, volume 47, 381-391.

[15] D. Mottet, Y. Guiard, T. Ferrand, and R. J. Bootsma, "Twohanded performance of a rhythmical Fitts task by individuals and dyads," Journal of Experimental Psychology: Human Perception and Performance, 2001, Vol. 27, No. 6, pp. 12751286.
[16] J. Accot and S. Zhai, "Beyond Fitts' law: Models for trajectorybased HCI tasks," Proceedings of the CHI 97, 1997, pp. 295302.

[17] J. C. A. Looser, "3D games as motivation in Fitts' law experiments", unpublished.

[18] K. Hinckley, E. Cutrell, S. Bathiche, and T. Muss, "Quantitative analysis of scrolling techniques," Conference on Human Factors in Computer Systems, pp. 65-72, 2002.

[19] S. Zhai, S. Conversy, M. Beaudouin-Lafon, Y. Guiard, "Human on-line response to target expansion," Proceedings of CHI 2003, ACM Conference on Human Factors in Computing Systems, Fort Lauderdale, Florida, April 2003. pp. 177-184.

[20] K. Wachholder, "Beitrge zur Physiologie der willkrlichen Bewegung," Mitteilung, Einzelbewegungen, Pflugers, Arch 214, pp. 642-661.

[21] M. Hallett, B. Shahani, R. Young, "EMG analysis of stereotyped voluntary movements in man," Journal of Neurosurgy Psychiatry, 1975, Vol. 38, pp. 1154-1162.

[22] B. Hannaford, L. Stark, "Roles of the elements of the triphasic control signal," Experimental Neurology, 1985, Vol. 90, pp. 619-634.

[23] G.L. Gottlieb, D.M. Corcos, G.C. Agarwal, M.L. Latash, "Principles underlying single joint movement strategies," J.M. Winters, S.L.Y. Woo, "Multiple Muscle Systems," SpringerVerlag, New York, pp 236-250.

[24] G.L. Gottlieb, D.M. Corcos, G.C. Agarwal, "Organizing principles for single-joint movements. I. A speed insensitive strategy," Journal of Neurophysiology, 1989 Vol. 62, pp. 342357.

[25] G.L. Gottlieb, C.H. Chen, D.M. Corcos, "Nonlinear control of movement distance at the human elbow," Experimental Brain Research, 1996, Vol. 112, pp. 289-297.

[26] C.F. Ramos, S.S. Hacisalihzade, L.W. Stark, "Behavior space of a stretch reflex model and its implications for the neural control of voluntary movement," 1990, Medical and Biological Engineering \& Computing, 1990, Vol. 28, pp. 15-23.

[27] J. Prodoehl, G. Gottlieb, D. Corcos, "The neural control of single degree-of-freedom elbow movements: Effect of starting joint position," Experimental Brain Research, 2003, Vol. 153, pp. 7-15.

[28] A. Karniel, R. Meir, G.F. Inbar, "Exploiting the Virtue of Redundancy," International Joint Conference on Neural Networks, Washington, DC, 1999.

[29] F. Lacquaniti, C. Maioli, "Distributed control of limb position and force," Requin GESaJ (ed) Tutorials in Motor Behavior II, Elsevier Science Publishers, New York, 1992, pp. 31-54.

[30] J.L. Patton, P. Elkins, "Training with a bimanual-grasp beneficially influences single limb performance," Society for Neuroscience, Orlando, FL, USA, 2002.

[31] S.S. Shergill, P.M. Bays, C.D. Frith, and D.M. Wolpert, "Two Eyes for an Eye: The Neuroscience of Force Escalation," Science Magazine, July 11, 2003, Vol. 301, p. 187. 